\title{
Neutron/Gamma-Ray Discrimination through Measures of Fit
}

\author{
Moslem Amiri, Václav Přenosil, and František Cvachovec
}

\begin{abstract}
Statistical tests and their underlying measures of fit can be utilized to separate neutron/gamma-ray pulses in a mixed radiation field. In this article, first the application of a sample statistical test is explained. Fit measurement-based methods require true pulse shapes to be used as reference for discrimination. This requirement makes practical implementation of these methods difficult; typically another discrimination approach should be employed to capture samples of neutrons and gamma-rays before running the fit-based technique. In this article, we also propose a technique to eliminate this requirement. These approaches are applied to several sets of mixed neutron and gamma-ray pulses obtained through different digitizers using stilbene scintillator in order to analyze them and measure their discrimination quality.
\end{abstract}

Index Terms-Counter/discriminator, statistical test, measures of fit, neutron spectroscopy, organic scintillator.

\section{INTRODUCTION}

$\mathbf{O}$ RGANIC scintillation detectors are widely used for neutron detection and spectroscopy. The primary reaction producing neutron field and scattering reactions of neutrons with materials in the environment lead to the production of $\gamma$ ray as a background radiation. The main problem in neutron detection is the discrimination of neutrons from the $\gamma$-rays. A composite curve often comprises a fast and a slow component of scintillation. The long-lived slow component often reveals the nature of the particle striking the detector. This fact is usually used to separate different kinds of particles; this process is called pulse shape discrimination (PSD) [1]. PSD techniques are mainly used to discriminate neutrons from the $\gamma$-rays.

Among organic scintillators, stilbene and NE-213 are favored for neutron spectroscopy purposes; they have rather low light output per unit energy, but this light output induced by charged protons can be easily distinguished from electrons/photons. Hence, stilbene and NE-213 scintillators produce very good results using PSD methods.

Time-domain PSD methods are not computationally intensive, and hence are suitable for real-time applications. Classically, following analog PSD techniques were most often used for $\mathrm{n} / \gamma$-ray discrimination [2]:

1) rise-time inspection;

Manuscript received April 6, 2015. This work was supported by Technology Agency of the Czech Republic under contract No. TA01011383/2011.

M. Amiri is with the Faculty of Informatics, Masaryk University, Botanicka 68a, 60200 Brno, Czech Republic (e-mail: amiri@mail.muni.cz).

V. Prenosil is with the Faculty of Informatics, Masaryk University, Botanicka 68a, 60200 Brno, Czech Republic (e-mail: prenosil@fi.muni.cz).

F. Cvachovec is with the Faculty of Military Technology, University of Defence, Kounicova 156/65, 66210 Brno, Czech Republic (e-mail: frantisek.cvachovec@unob.cz).
2) zero-crossing method;

3) charge comparison.

Although analog techniques make acceptable $n / \gamma$-ray discrimination, availability of precise and fast digitizers and various PSD algorithms have made it possible to do a better discrimination of these radiations digitally. Among digital PSD methods, pulse rise-time algorithm and charge comparison are probably the most favorable ones.

Measures of fit of statistical tests can be easily used to discriminate neutrons and $\gamma$-rays. The underlying operations in these tests are generally the same. In this article, the application of a statistical test, namely Pearson's chi-squared test, is utilized to separate $\mathrm{n} / \gamma$-ray pulses in a mixed radiation field. Since fit measurement-based methods require two reference pulse shapes to be preliminarily available for discrimination, an update to the original implementation is introduced later in this article to eliminate this requirement. To obtain the sampled data of mixed neutron and $\gamma$-ray pulses, two differently-featured digitizers (explained in Section II) are used which differ mainly in their sampling rate and output quantization level resolution. Doing so, the effect of resolution and sampling frequency of the digitizers on the quality of the discrimination result for the methods discussed in this article could be found. Every experiment is carried out under the same experimental conditions, using 100,000 pulses of mixed neutron and photon signals. For this work, the field consists of mostly $\gamma$-rays and some neutrons.

A comparison among various techniques, applied to data obtained from the different digitizer types and settings, is done by using the Figure of Merit (FoM) for the $n / \gamma$-ray discrimination, defined as:

$$
F o M=\frac{S}{F W H M_{n}+F W H M_{\gamma}}
$$

where $S$ is the separation between the peaks of the two events, $F W H M_{\gamma}$ is the full-width half-maximum (FWHM) of the spread of events classified as $\gamma$-rays and $F W H M_{n}$ is the FWHM of the spread in the neutron peak [3]. FWHMs are calculated using the Gaussian fits to the neutron and $\gamma$-ray events on experimental distribution plot.

\section{EXPERIMENTAL SETUP}

For this work, stilbene scintillation detector was used with $45 \times 45$ crystal, and the neutron-gamma radiation source used was $252 \mathrm{Cf}(\mathrm{sf})$. A typical scintillation detector consists of a scintillator and a photomultiplier. The latter is employed to change weak light signals impinging to photocathode (generated by the scintillator) into electric impulses. We used the photomultiplier RCA7265 [4] throughout these experiments. 


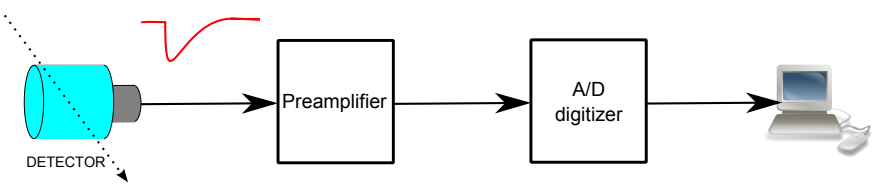

Fig. 1. Block diagram of a digital two-parameter analyzer.

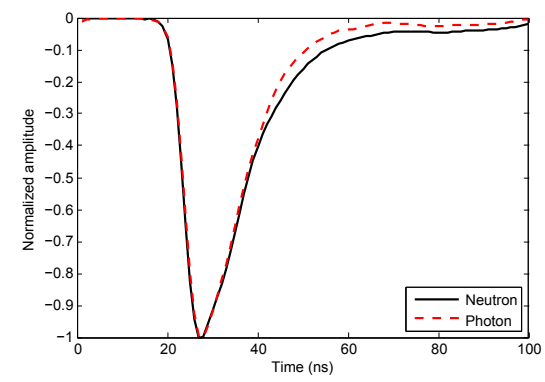

Fig. 2. Comparison of a sample smoothed neutron with a sample smoothed photon. These signals are obtained from the stilbene scintillator.

The block diagram of our digital apparatus is shown in Fig. 1.

A preamplifier is selected so as to match the detector output impedance. Two variants of the anode load resistance were tested in conjunction with the organic scintillation detectors. In the first variant, a load resistance of $40 k \Omega$ was used. A preamplifier matched it to the coaxial cable whose characteristic impedance was $50 \Omega$. In this case, the different waveforms of the neutron and photon pulses can be detected in the voltage pulse leading edge. If the magnitude of the load resistance is selected to be close to the characteristic impedance of the coaxial cable, which is $50 \Omega$, the different shapes of the neutron/photon pulses will appear to take effect during the decay time. In this case, no preamplifier is necessary. The latter option was employed here.

Two commercially available Agilent digitizers were used to digitize the output pulses: Acqiris DP210 with 8-bit resolution and set at 1 and $2 \mathrm{GS} / \mathrm{s}$, and Acqiris DC440 with 12-bit resolution and set at 250 and $420 \mathrm{MS} / \mathrm{s}$. While real-time digitizers are also employed in industry today, we used these specific commercial digitizers to study the effects of their various data resolution and sampling frequency features on digital processing.

\section{Neutron And Photon Signals}

A sample smoothed neutron is compared with a sample smoothed photon pulse in Fig. 2. These signals are obtained from the stilbene scintillator. As seen in this Figure, these signals are composed of a leading and a trailing edge. The leading edges could not be exploited for discrimination purposes. On the other hand, the trailing edge of the neutron signal has higher rise time than that of the photon signal. This property could be used to separate these two radiations.

An innovative discrimination approach is to remove the similar segments of the two signal types and apply the technique only to the differing segments.

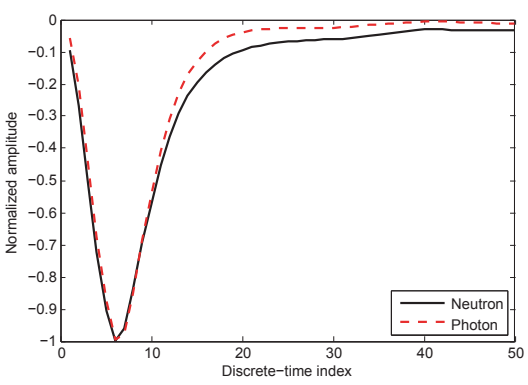

Fig. 3. Segments of neutron and $\gamma$-ray true pulse shapes, obtained by averaging multiple normalized neutron and $\gamma$-ray pulses. The original pulses are obtained from DC440 digitizer (12-bit resolution, $420 \mathrm{MS} / \mathrm{s}$ ). The same segment of every unknown pulse from the data set will be used for the measurements.

\section{MEASURES OF Fit}

Measures of fit of statistical tests can be employed to discriminate neutrons and $\gamma$-rays. The underlying operations in these tests are generally the same. In [5], [6], a similar approach, called "model pulse algorithm", is adopted. In the following, we propose a simpler and more practical approach to separating radiations.

First, we construct two normalized reference pulse shapes, called true pulse shapes, one for neutrons and the other for $\gamma$ rays. The $\gamma$-ray true pulse shape can be obtained by averaging some normalized signals captured from different pure $\gamma$ ray sources, and the neutron true pulse shape is obtained by averaging some normalized neutron pulses captured by discrimination through some method, e.g. integration method (the latter approach can be used to build the $\gamma$-ray true pulse shape too). For this work, the starting point of the two normalized true pulse shapes is where the amplitude of the leading edge is $10 \%$ fraction of peak-amplitude, and the end point is some specific time after the starting point such that this interval includes the differing tail segment between neutrons and $\gamma$-rays. The same approach used to cut a segment from the true pulse shapes will also be used to cut a segment from each normalized unknown pulse from a mixed $n / \gamma$-ray field. Although the starting point could be set to be the peak of a pulse since the leading edges of neutron and $\gamma$-ray pulses almost map on each other, the inclusion of the leading edge (from $10 \%$ level to the peak) will make the calculations more precise. This segment is shown in Fig. 3, and used throughout our experiments.

Second, a statistical test is applied to separate $n / \gamma$-ray pulses in a mixed radiation field. In this article, Pearson's chi-squared test is utilized as an example statistical test. By applying the test, two values are obtained by measuring the fit of the captured pulse and the two true pulse shapes $\left(\chi_{n}^{2}, \chi_{\gamma}^{2}\right)$. The measurements are done only within a specific segment of the pulses and the true pulse shapes, as explained above. The lower resulting value assigns the particle type. This approach can be extended to the other statistical tests available. The distribution of the $\left(\chi_{n}^{2}, \chi_{\gamma}^{2}\right)$ values provides an excellent discrimination of neutrons and $\gamma$-rays.

The equation to implement Pearson's chi-squared test for 


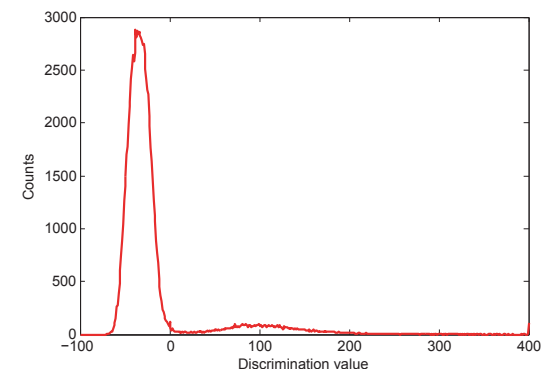

Fig. 4. Discrimination of $\gamma$-ray and neutron signals, applying the statistical test approach. The pulses are obtained using DP210 digitizer (8-bit resolution, $1 \mathrm{GS} / \mathrm{s})$

TABLE I

FOMS AND COUNTS OF THE PULSES OBTAINED FROM DC440 AND DP210 DIGITIZERS UNDER DIFFERENT SAMPLING RATES. STATISTICAL TEST APPROACH IS APPLIED TO THE DATA.

\begin{tabular}{l|lll}
\hline Data format & FoM & $\begin{array}{l}\text { Neutron } \\
\text { counts }\end{array}$ & $\begin{array}{l}\text { Photon } \\
\text { counts }\end{array}$ \\
\hline 12-bit, 250 MS/s & 1.10 & 9876 & 90124 \\
\hline 12-bit, 420 MS/s & 1.02 & 9795 & 90205 \\
\hline 8-bit, 1 GS/s & 1.02 & 10076 & 89924 \\
\hline 8-bit, 2 GS/s & 1.09 & 9920 & 90080 \\
\hline
\end{tabular}

$\mathrm{n} / \gamma$-ray discrimination is:

$$
\chi^{2}=\sum_{i=1}^{n} \frac{\left(O_{i}-E_{i}\right)^{2}}{E_{i}}
$$

where $O_{i}$ is the observed value and $E_{i}$ is the expected value. For discrimination application, $O_{i}$ and $E_{i}$ are the corresponding points in the sequences of captured pulse and the true pulse shapes respectively. Two $\chi^{2}$ values are calculated; one between the input pulse and the neutron true pulse $\left(\chi_{n}^{2}\right)$, and the other between the input pulse and the $\gamma$-ray true pulse $\left(\chi_{\gamma}^{2}\right)$. The smaller value between these two will determine the input pulse type. Hence this method could be used to count the number of pulses from each category. The subtraction of the two resulting $\chi^{2}$ values for each input pulse could be used to calculate the FoM for that set of pulse data. This subtraction will scatter neutrons and $\gamma$-rays on two different sides of the zero base line of the plot. Figure 4 illustrates the discrimination plot for the case of DP210 digitizer (8-bit resolution) with $1 \mathrm{GS} / \mathrm{s}$ sampling rate. As seen in this Figure, neutrons have positive and $\gamma$-rays have negative discrimination values in this experiment.

FoMs and $\mathrm{n} / \gamma$-ray counts for various data sets with different resolutions and frequency rates are shown in Tab. I. The results are solid; this method discriminates well irrespective of the digitizer features.

\section{IMPROVED ALGORITHM}

In the preceding technique, multiple detected neutrons and $\gamma$-rays are needed to build the fixed true pulse shapes to be used throughout the experiment. This requires the application of another discrimination technique prior to the statistical test in order to build the true pulse shapes. It is possible to build the true pulse shapes without the preliminary separation technique application, as will be explained in this section.

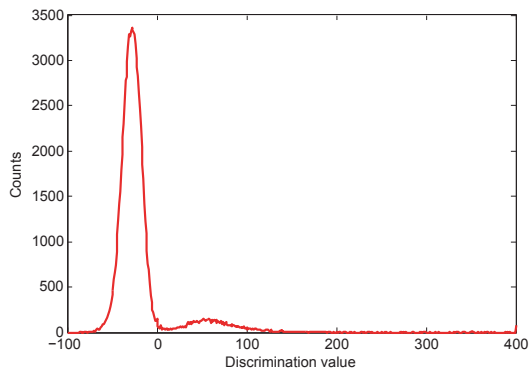

Fig. 5. Discrimination of $\gamma$-ray and neutron signals, applying the improved statistical test approach. The pulses are obtained using DP210 digitizer (8-bit resolution, $1 \mathrm{GS} / \mathrm{s})$.

A simple method to build the two reference pulses is to pick two random pulses (e.g. the first two pulses at the beginning of the experiment) regarding each as representing a true pulse shape. The identity of these pulses are unknown and they may be even of the same type. The statistical test technique is applied to the rest of the pulses, using the two selected reference pulses. Every input pulse is categorized under one of the reference pulses which produces the lower resulting value, and the reference pulse is updated with that input pulse. Using this strategy, one reference sequence will be gradually moved toward the neutron and the other toward a $\gamma$-ray pulse shape.

Instead of the initial random selection of the two reference pulses, a better method is to average multiple random pulses and build up two new sequences by roughly shaping the resulting averaged sequence, once as neutron and once as $\gamma$ ray (it is not necessary to be precise). Then the statistical test approach is applied to every pulse, using the two rough references. The references are updated as the experiment goes on.

Using either of the methods explained above in this section, the pulses will be divided and grouped with either of the two reference sequences. If not detected, at the end of the experiment, when the reference pulses are completely refined and shaped, a simple test will reveal the identity of the two reference sequences, hence the identity of the pulses grouped with each reference pulse. For example, since the reference and input pulses are normalized throughout the experiment, if the samples of each reference pulse are summed up, the one with the lower sum will be $\gamma$-ray and the other one will be neutron. Fig. 5 illustrates the experimental distribution plot of neutrons and $\gamma$-rays for the data obtained from DP210 digitizer with 8-bit resolution and set at $1 \mathrm{GS} / \mathrm{s}$ frequency rate, when this method is applied.

FoMs and $\mathrm{n} / \gamma$-ray counts for various data sets with different resolutions and frequency rates are shown in Tab. II. The same data sets used for the experiments of Section IV are used here for comparison purposes. Although this method does not require the true pulse shapes prior to the experiment, it does not produce acceptable FoMs or accurate pulse counts. The lowest discrimination quality and the most inaccurate pulse count is when the sampling rate of the digitizer is low. 
TABLE II

FoMs AND COUNTS OF THE PULSES OBTAINED FROM DC440 AND DP210 DIGITIZERS UNDER DIFFERENT SAMPLING RATES. IMPROVED STATISTICAL TEST APPROACH IS APPLIED TO THE DATA.

\begin{tabular}{l|lll}
\hline Data format & FoM & $\begin{array}{l}\text { Neutron } \\
\text { counts }\end{array}$ & $\begin{array}{l}\text { Photon } \\
\text { counts }\end{array}$ \\
\hline 12-bit, 250 MS/s & 0.78 & 22780 & 77220 \\
\hline 12-bit, 420 MS/s & 1.03 & 9496 & 90504 \\
\hline 8-bit, 1 GS/s & 0.98 & 9817 & 90183 \\
\hline 8-bit, 2 GS/s & 0.84 & 10289 & 89711 \\
\hline
\end{tabular}

\section{DISCUSSION}

Two important factors affecting the FoM of a discrimination method are resolution and sampling rate of the digitizer. According to Nyquist criterion, the sampling rate must be greater than twice the bandwidth of continuous digitizer input signal. The FFT of the recorded neutron and photon signals indicates frequency components up to $100 \mathrm{MHz}$ [7]. Therefore, the minimum necessary sampling frequency for neutron and photon signals is about $200 \mathrm{MS} / \mathrm{s}$. The exact impact of the sampling rate on the separation quality of a specific method depends on how the method functions, and estimation of this effect can be involved. For the approaches introduced in this article, as Tabs. I and II show, increasing from the low sampling rate of $250 \mathrm{MHz}$ (which is close to the minimum $200 \mathrm{MHz}$ required) to $420 \mathrm{MHz}$ or increasing from the high sampling rate of $1 \mathrm{GHz}$ to $2 \mathrm{GHz}$ does not necessarily improve the FoM.

The factor with a greater impact on discrimination quality is digitizer resolution. The process of converting a discrete-time continuous-amplitude signal into a digital signal by expressing each sample value as a finite number of digits is called quantization. The resolution (or quantization step size) is the distance between two successive quantization levels. The error introduced in representing the continuous-valued signal by a finite set of discrete value levels is called quantization error or quantization noise. The quality of the digitizer output could be measured by signal-to-quantization noise ratio (SQNR). Since quantization errors of neutron and photon signals are almost uniformly distributed over the quantization interval, the following well-known equation [8] reliably estimates the quality of a $b$-bit digitizer output:

$$
\operatorname{SQNR}(d B)=1.76+6.02 b
$$

Eq. 3 implies that SQNR increases approximately $6 \mathrm{~dB}$ for every bit added to the digitizer word length. This relationship gives the number of bits required by an application to assure a given signal-to-noise ratio.

In order to verify the performance of the methods introduced in this article, we apply PGA method to the same pulse data sets as used for the methods in this paper. PGA method, introduced in [3], is recognized as an efficient $\mathrm{n} / \gamma$-ray discrimination method with a high FoM. The slower decay of the light function of a scintillator for a neutron interaction than that for a $\gamma$-ray interaction is exploited in this method. The gradient between the peak amplitude and the amplitude a specified time after the peak amplitude (called the discrimination amplitude) on the trailing edge of the pulses are compared and used

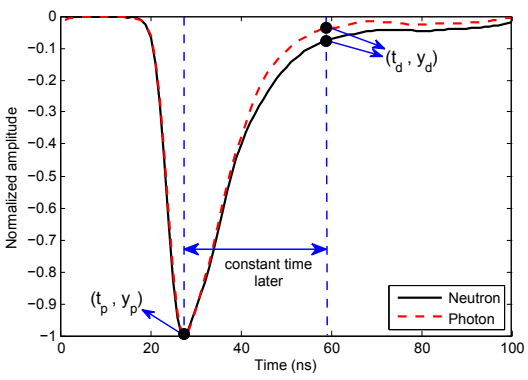

Fig. 6. The points on smoothed neutron and photon signals used in PGA discrimination method.

TABLE III

FOMS OF PGA METHOD FOR THE PULSES OBTAINED FROM VARIOUS DIGITIZERS

\begin{tabular}{l|llll}
\hline Digitizer & 8-bit, 1 GS & 8-bit, 2 GS & 12-bit, 250 MS & 12-bit, 420 MS \\
\hline FoM & 0.88 & 0.91 & 0.94 & 1.00
\end{tabular}

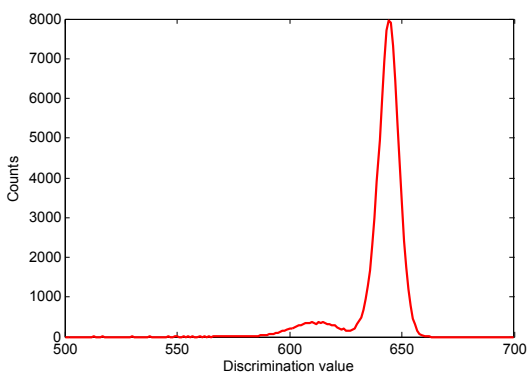

Fig. 7. Discrimination of photon and neutron signals, applying PGA method. The pulses are obtained using DC440 digitizer (12-bit resolution, $420 \mathrm{MS} / \mathrm{s}$ ).

as the discrimination factor. Fig. 6 illustrates the peak and discrimination amplitudes on neutron and photon signals. The gradient is calculated using

$$
m=\frac{\Delta y}{\Delta t}=\frac{\left(y_{p}-y_{d}\right)}{\left(t_{p}-t_{d}\right)}
$$

where $m, y_{p}, y_{d}, t_{p}$, and $t_{d}$ are the gradient, the peak amplitude (which is a constant for normalized pulses), the discrimination amplitude, the time of peak amplitude occurrence, and the time of discrimination amplitude occurrence, respectively. For this work, we used some training pulses to locate the best discrimination amplitude, which occurred about $36 \mathrm{~ns}$ after the peak of the pulse. In general, the optimal timing for the discrimination amplitude which makes the highest difference between the two radiation types is dependent on the scintillator properties and also on the PMT. The FoMs obtained are listed in Tab. III. A comparison shows that the novel methods introduced here are either better or at least have the same discrimination quality as the PGA method does. Fig. 7 shows the best discrimination plot obtained by PGA method.

\section{CONCLUSION}

In this article, we first introduced $\mathrm{n} / \gamma$-ray discrimination through measures of fit, particularly using Pearson's chisquared test. This approach counts and discriminates the pulses in a mixed environment very efficiently, however, it requires sample neutron and $\gamma$-ray pulses in advance of the experiment 
run to build the true pulse shapes. This requirement leads to the employment of another technique prior to the statistical test method to provide proper reference sequences. Since this may not be practically acceptable, an improvement to the original statistical test method is made in this article to build the true pulse shapes during run time. The true pulse shapes are gradually updated and used as the reference throughout the experiment. Two digitizers, each featuring a different resolution and each set at two different sampling rates, were used to observe the reaction of the methods to the data sampling conditions. The original approach (requiring true pulse shapes prior to the experiment) provides promising results while the improved approach is inefficient specially when applied to the data sampled at low rates.

\section{REFERENCES}

[1] G. F. Knoll, Radiation Detection and Measurement, 3rd ed. New York: Wiley, 2000, ch. 8, p. 230.

[2] G. Ranucci, "An analytical approach to the evaluation of the pulse shape discrimination properties of scintillators," Nuclear Instruments and Methods in Physics Research Section A: Accelerators, Spectrometers, Detectors and Associated Equipment, vol. 354, no. 2-3, pp. 389 - 399, 1995. [Online]. Available: http://www.sciencedirect.com/science/article/pii/0168900294008868

[3] B. Mellow, M. Aspinall, R. Mackin, M. Joyce, and A. Peyton, "Digital discrimination of neutrons and g-rays in liquid scintillators using pulse gradient analysis," Nuclear Instruments and Methods in Physics Research Section A, vol. 578, no. 1, pp. 191-197, 2007.

[4] "Rca 7265 photomultiplier tube 2" 14-stage s-20," http://www.hofstragroup.com/product/rca-7265-photomultiplier-tube2-14-stage-s-20/, accessed: 2014-07-15.

[5] S. Marrone, D. Cano-Ott, N. Colonna, C. Domingo, F. Gramegna, E. Gonzalez, F. Gunsing, M. Heil, F. Käppeler, P. Mastinu, P. Milazzo, T. Papaevangelou, P. Pavlopoulos, R. Plag, R. Reifarth, G. Tagliente, J. Tain, and K. Wisshak, "Pulse shape analysis of liquid scintillators for neutron studies," Nuclear Instruments and Methods in Physics Research Section A: Accelerators, Spectrometers, Detectors and Associated Equipment, vol. 490, no. 1-2, pp. 299-307, 2002. [Online]. Available: http://www.sciencedirect.com/science/article/pii/S016890020201063X

[6] C. Guerrero, D. Cano-Ott, M. Fernández-Ordóñez, E. GonzálezRomero, T. Martínez, and D. Villamarín, "Analysis of the BC501A neutron detector signals using the true pulse shape," Nuclear Instruments and Methods in Physics Research Section A: Accelerators, Spectrometers, Detectors and Associated Equipment, vol. 597, no. 2-3, pp. 212-218, 2008. [Online]. Available: http://www.sciencedirect.com/science/article/pii/S0168900208014113

[7] F. Belli, B. Esposito, D. Marocco, and M. Riva, "A study on the pulse height resolution of organic scintillator digitized pulses," Fusion Engineering and Design, vol. 88, no. 68, pp. 1271 - 1275, 2013, proceedings of the 27th Symposium On Fusion Technology (SOFT-27); Liége, Belgium, September 24-28, 2012. [Online]. Available: http://www.sciencedirect.com/science/article/pii/S092037961200587X

[8] J. G. Proakis and D. G. Manolakis, Digital Signal Processing, Fourth Edition. Upper Saddle River, New Jersey 07458: Prentice Hall, 2006. 